\title{
Heartbeat: Coronary heart disease, obesity, smoking and long-lasting psychological distress
}

doi:10.1136/heartjnl-2017-311592

The importance of depression and psychological distress as a risk factor for coronary heart disease (CHD), is widely recognised, particularly in post-acute myocardial infarction patients. In this issue of Heart, Virtanen and colleagues ${ }^{1}$ report an original contribution to this field studying the long-term association of CHD and cardiovascular risk factors with levels of psychological distress over the patient's lifetime, including symptoms of depression and anxiety. Using seven assessments of psychological distress over a 21-year follow-up of 6890 participants from the Whitehall II cohort study, the authors used group-based trajectory models (GBTM) to identify clusters of individuals (trajectory groups) with a similar trajectory over time (figure 1). The participants were grouped according to their trajectories as 'persistently low' (69\% of the participants), 'persistently intermediate' (13\%), 'intermediate to low' (12\%), and 'persistently high' (7\%) psychological distress. Prevalent CHD was associated with a approximately 1.9-fold, smoking with a 1.6 -fold and obesity with a 1.5 -fold likelihood of a persistently high psychological distress trajectory. After discussing the mechanisms related to this association, the authors concluded that 'clinicians should be aware of the potential longlasting associations of CHD, obesity and smoking as risk factors for prolonged psychological distress'.

In the companion editorial, Barreto and Giatti' $^{2}$ stated that 'expanding the knowledge on the long-term bidirectional relationship between depressive symptoms and coronary heart disease (CHD) is key for decreasing the excess morbidity and mortality associated with both conditions, and for reducing healthcare costs'. For the physician, they remind that 'screening and treating mental health problems of individuals with CHD is not a one-time approach, but a continuous goal'.

In adults with tetralogy of Fallot (TOF), a prolonged QRS duration is

Division of Cardiology, University of Washington, Seattle, USA

Correspondence to Professor Catherine M Otto, Division of Cardiology, University of Washington, Seattle, Washington 98195, USA; cmotto@uw.edu

\section{Antonio Luiz Ribeiro, Satoshi Nakatani and Catherine M Otto}

a known predictor of adverse clinical outcomes. In this issue of Heart, Bokma and colleagues $^{3}$ studied the predictive value of QRS fragmentation, defined on the finding in 2 contiguous leads of 2 or more notches in the QRS complex in the absence of bundle branch block or as 3 or more $\mathrm{R}$ wave notches when right bundle branch block was present. In 794 TOF patients overall 10-year survival in the $52 \%$ with no QRS fragmentation was $98 \%$ compared with $81 \%$ in the $16 \%$ of patients with severe QRS fragmentation. Multivariable Cox regression analysis showed that QRS fragmentation (but not QRS duration) was independently predictive for mortality and ventricular arrhythmias (figure 2).

In the accompanying editorial, ${ }^{4}$ Heng and Gatzoulis comment that "Identification of at-risk patients with repaired tetralogy of Fallot and QRS fragmentation may better guide the choice and rationalisation of onward investigations. The predictive value of QRS fragmentation may be further enhanced by combining this electrical marker with cardiac functional and structural data from imaging such as echocardiography and MRI in future work."

Speckle tracking echocardiography can assess myocardial strain which reflects precise myocardial function. Many studies have shown the superiority of myocardial strain over conventional functional parameters, such as ejection fraction, for prediction of clinical outcomes in various types of cardiovascular disease. However, while echocardiography excels at assessment of function, it is not suitable for evaluation of tissue pathology. Cardiac magnetic resonance (CMR) has the advantage that the presence and extent of myocardial fibrosis can be visualised using late gadolinium enhancement (LGE). Therefore, by using both modalities in a mutually complementary manner, prognosis of cardiovascular diseases may be better predicted. Chimura and colleagues $^{5}$ report that combination of global longitudinal strain (GLS) and LGE were useful for risk stratification and prognostic assessment in 179 patients with non-ischaemic dilated cardiomyopathy. They found that GLS is associated with left ventricular reverse remodelling after optimal medical therapy whereas LGE is associated with life-threatening arrhythmias. The combination of GLS and LGE was most predictive of longterm outcomes (figure 3). Given the rapid proliferation of new imaging modalities, we need to carefully assess the value and limitations of each modality in clinical practice to use imaging effectively and efficiently. As in the study by Chimura
Figure 1 Trajectories of psychological distress symptoms (General Health Questionnaire-30) among the Whitehall II study participants 


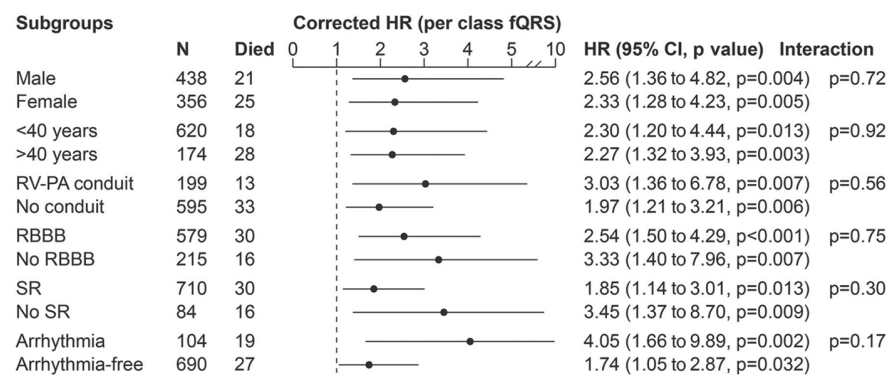

Figure 2 Predictive value of QRS fragmentation within subgroups. Corrected HR (for age, previous pacemaker and previous shunt) with $95 \% \mathrm{Cl}$ of extent of $\mathrm{fQRS} /$ class within different subgroups. p Values for interaction terms between subgroups and fQRS extent are displayed. Previous arrhythmia subgroup included all patients with either clinical ventricular arrhythmia or supraventricular tachycardia before CONCOR inclusion. RV-PA conduit subgroup included all patients with conduit-type repair or previous pulmonary valve replacement. fQRS, fragmented QRS complexes; $\mathrm{PA}$, pulmonary artery; $\mathrm{RBBB}$, right bundle branch block; RV, right ventricle; $\mathrm{SR}$, sinus rhythm.
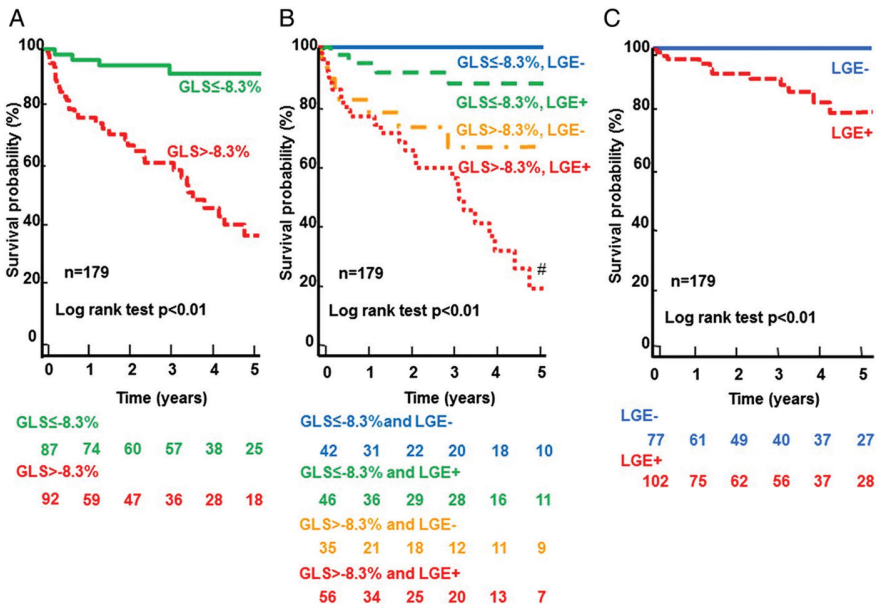

Figure 3 Kaplan-Meier analysis according to left ventricular global longitudinal strain (GLS) and late gadolinium enhancement (LGE). (A)Patients with reduced GLS (>-8.3\%: red line) had a worse long-term outcome than those with preserved GLS ( $\leq-8.3 \%$ : green line). (B) In the paMents with reduced GLS(-8.3\%), the presence of LGE was associated with adverse events. \#p $<0.05$ vs patients with GLS $>-8.3 \%$ and LGE-. (C) Freedom from life-threatening ventricular arrhythmias according to LGE. The presence of LGE was strongly associated with life-threatening ventricular arrhythmias. There were no events in patients without LGE (LGE-).
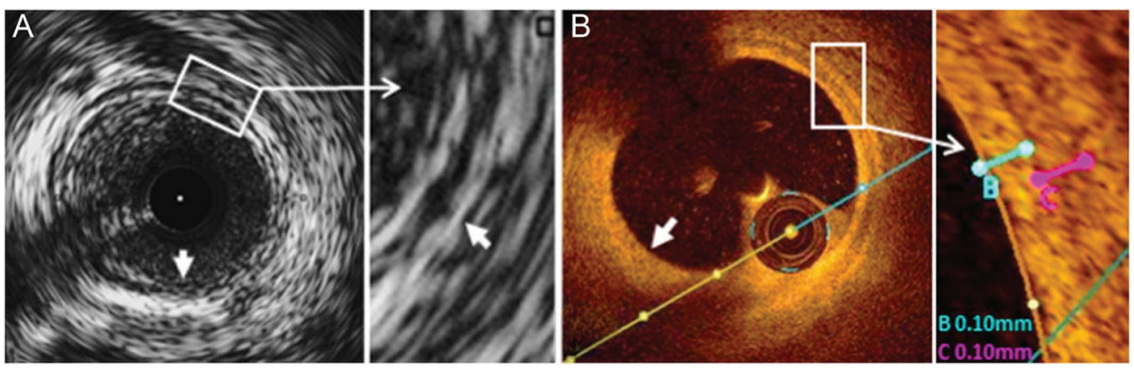

Figure 4 Optical coherence tomography (OCT)/intravascular ultrasound (IVUS) characteristics of normal and pathological vessel wall. (A, B) Intimal thickening and fibrotic eccentric plaque: IVUS (A) and OCT (B) cross section in a distal segment showing eccentric fibrosis (arrow) associated with loss of the three-layer aspect that can still be recognised in the opposite quadrants as displayed in the magnified boxes (on the right). (A) IVUS resolution does not allow to distinguish the intimal leading edge in a normal vessel, but the intima-media complex is well delineated in the presence of intimal thickening; the external elastic membranemedia interface is best recognised with IVUS (arrow). and colleagues, we may find that combinations of imaging parameters provide more useful clinical information than either alone: one plus one may be larger than two.

The Education in Heart article in this issue $^{6}$ focuses on intravascular ultrasound with a review of physical principles, operational modalities, image interpretation and methodology for measuring vessel diameters, areas and derived indices (figure 4). In addition, an update is provided on the evolving clinical applications of intravascular ultrasound and optical coherence tomography, particular after coronary stent implantation.

Check out the Image Challenge! Can you make the correct diagnosis based on the clinical presentation and cardiac magnetic resonance images?

Competing interests None declared.

Provenance and peer review Commissioned; internally peer reviewed.

(C) Article author(s) (or their employer(s) unless otherwise stated in the text of the article) 2017. All rights reserved. No commercial use is permitted unless otherwise expressly granted.

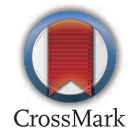

To cite Otto CM. Heart 2017;103:643-644.

Heart 2017;103:643-644.

doi:10.1136/heartjnl-2017-311592

\section{REFERENCES}

1 Virtanen M, Elovainio M, Josefsson K, et al. Coronary heart disease and risk factors as predictors of trajectories of psychological distress from midlife to old age. Heart 2017:103:659-65.

2 Barreto SM, Giatti L. Psychological distress: a longlasting matter of the heart. Heart 2017;103:649-50.

3 Bokma JP, Winter MM, Vehmeijer JT, et al. QRS fragmentation is superior to QRS duration in predicting mortality in adults with tetralogy of fallot. Heart 2017;103:666-71.

4 Heng EL, Gatzoulis MA. QRS fragmentation in tetralogy of fallot: clinical utility and risk prediction. Heart 2017; 103:645-6.

5 Chimura M, Onishi T, Tsukishiro Y, et al. Longitudinal strain combined with delayed-enhancement magnetic resonance improves risk stratification in patients with dilated cardiomyopathy. Heart 2017;103:679-86.

6 Giavarini A, Kilic ID, Redondo Diéguez A, et al. Intracoronary imaging. Heart 2017;103:708-25.

7 Moss AJ, Dweck MR, O'Kane PD. A 56-year-old woman with breathlessness. Heart 2017;103:686. 